Revue d'histoire de l'enfance « irrégulière »

Le Temps de l'histoire

16 | 2014

La part scolaire : jeunesse irrégulière et école $\left(\mathrm{XIX}^{\mathrm{e}}\right.$ $\mathrm{XX}$ siècles)

\title{
Un adolescent condamné à mort en 1975
}

Being a teenager sentenced to death in France in 1975

Jean-Claude Vimont

\section{(2) OpenEdition}

1 Journals

Édition électronique

URL : https://journals.openedition.org/rhei/3676

DOI : $10.4000 /$ rhei.3676

ISBN : 978-2-7535-4019-4

ISSN : $1777-540 \mathrm{X}$

Éditeur

Presses universitaires de Rennes

Édition imprimée

Date de publication : 30 octobre 2014

Pagination : 159-190

ISBN : 978-2-7535-3558-9

ISSN : $1287-2431$

Référence électronique

Jean-Claude Vimont, « Un adolescent condamné à mort en 1975 », Revue d'histoire de l'enfance «

irrégulière » [En ligne], 16 | 2014, mis en ligne le 30 octobre 2016, consulté le 08 septembre 2021. URL : http://journals.openedition.org/rhei/3676 ; DOI : https://doi.org/10.4000/rhei.3676

Ce document a été généré automatiquement le 8 septembre 2021.

(C) PUR 


\title{
Un adolescent condamné à mort en
} 1975

\author{
Being a teenager sentenced to death in France in 1975
}

Jean-Claude Vimont

\section{NOTE DE L'ÉDITEUR}

Cet article est la version légèrement remaniée et écourtée d'un texte initialement paru sous forme électronique dans Criminocorpus la revue [http://criminocorpus.revues.org/ 2673]. Au regard de l'intérêt de ce travail, le comité de rédaction avait souhaité offrir aux lecteurs de la Revue d'histoire de l'enfance « irrégulière » une édition « papier » de l'histoire du dernier mineur condamné à mort en France.

1 Ces dernières années, l'ordonnance de 1945 qui organise depuis plus d'un demi-siècle la justice des mineurs, coupables de délits et de crimes, a encore été remise en cause. Les principes d'atténuation de la responsabilité et des peines ont été contestés. Des voix se sont fait entendre pour abaisser les âges en deçà desquels les jeunes étaient déclarés irresponsables. Le primat de l'éducatif sur le répressif, fondement essentiel de l'Éducation surveillée, puis de la Protection judiciaire de la jeunesse, fut lui aussi bousculé. Des établissements pénitentiaires pour mineurs furent construits tout comme des centres éducatifs fermés. De jeunes guetteurs facilitant le trafic de drogue dans les cités et des pickpockets d'origine roumaine arrêtés dans le métropolitain posent la question de la frontière entre l'enfance et l'adolescence, de l'éventuel abaissement du passage d'une époque à une autre. C'est du moins l'opinion d'une fraction du public.

En novembre 1974, les quatre auteurs de l'assassinat de Madame Simone Boursier à Liancourt sont des adolescents. Yannick, Michel, Patrick et Bruno ont entre seize et dixhuit ans. Le Nouvel Observateur, un an plus tard, fit sa couverture avec le titre «Un enfant condamné à mort ", lorsque Bruno T., l'un des quatre jeunes gens fut frappé de ce verdict le 3 octobre 19751. «Faut-il tuer les jeunes?» s'interrogea Antoine Marcilhacy, dans un ouvrage publié aux lendemains de la grâce octroyée par le 
président Valéry Giscard d'Estaing le 11 février $1976^{2}$. Des enfants ? Des adolescents? Des jeunes? Les commentaires étaient très influencés par une grille de lecture fondée sur une supposée opposition entre les générations, une lutte entre la jeunesse et l'univers des vieux. Pour une frange de la presse favorable à l'exécution du mineur, il ne s'agissait que de monstres. Cela figurait en toutes lettres en couverture de l'hebdomadaire d'extrême droite Minute.

\section{Des gamins dans un foyer d'action éducative à Beauvais $^{3}$}

3 Yannick et Michel sont issus de familles nombreuses. Ils sont huit frères et sœurs chez Yannick, l'âné de la fratrie, à Amiens. Deux d'entre eux souffrent de handicaps mentaux, tout comme la maman. Le père est ramoneur. Michel est le cinquième de neuf enfants. Son père souffre d'une grave invalidité cardiaque. Il boit et est violent. Les disputes, les violences sont à l'arrière-plan de son enfance. Ce sont les parents qui se déchirent, mais aussi les bagarres avec les copains. Un éducateur qualifiait son entourage de «milieu pathogène et inéducatif ». Michel avait l'impression d'être le souffre-douleur de son père, le «mal aimé » de la famille. Le milieu familial de Patrick n'avait rien à envier aux deux précédents. La famille était très pauvre. Son père était également alcoolique et colérique. Il venait de mourir d'une cirrhose. Patrick n'avait guère connu sa mère. Elle avait été internée dans l'établissement psychiatrique de Clermont-de-l'Oise alors qu'il n'avait que six ans. Si ces jeunes sont placés dans le foyer de Beauvais, c'est aussi parce que les relations familiales sont tendues et insupportables. Yannick, vers dix-sept ans ne s'entend pas avec son père. Sa mère l'incite à s'engager dans l'armée. Il demande lui-même son placement au foyer de Beauvais pour quitter une ambiance familiale délétère. Le père de Patrick s'était mis en ménage avec une femme après l'internement de son épouse. Le jeune ne s'entendait pas avec cette "belle-mère", cette marâtre comme on disait autrefois. Les rapports d'autorité au sein de la famille étaient complexes. Pour se venger de cette femme qui menaçait de le « placer », Patrick viola sa sœur !

Bruno était lui aussi, comme deux de ses camarades, issu d'un père alcoolique qu'il connut à peine puisque le géniteur déserta le foyer familial. Sa mère était aidesoignante. Les parents avaient divorcé en 1960. Né le 9 avril 1957 dans le Pas-de-Calais, il n'avait alors que trois ans. De l'âge de trois ans à sept ans, Bruno fut élevé dans un orphelinat. Il retourna ensuite vivre chez sa mère à Liancourt, jusqu'à l'âge de seize ans, date d'un nouveau déménagement. Sa mère avait refait sa vie et était repartie s'installer dans le Pas-de-Calais. Elle avait un nouvel homme dans sa vie. Bruno ne s'entendait pas avec son «beau-père ». Bruno avait à peine seize ans lorsqu'il arriva dans le Pas-de-Calais. Il ne fut pas inscrit dans un collège d'enseignement technique et commença à aller de " petits boulots en petits boulots ». Les éducateurs successifs qui se penchèrent sur son cas ne purent que constater sa «difficulté à structurer sa personnalité ", à la suite d'autant de ruptures familiales, de foyers successifs.

Ils ont tous eu un parcours scolaire chaotique. Michel a eu des résultats médiocres à l'école primaire, puis au collège puis en CET. À plusieurs reprises, il fugue et se réfugie dans la boisson. À seize ans, ses débuts professionnels dans la mécanique automobile sont difficiles. Yannick a connu les classes de perfectionnement d'Amiens. Il a souvent entendu dans son entourage l'expression «débile mental » à son propos. À l'âge de 
quinze ans, il fut placé dans un institut médico-pédagogique, à la suite d'une hospitalisation de deux mois dans un hôpital psychiatrique. Il fugua pour retrouver sa famille, ses sept frères et sœurs. L'année suivante, il ne demeura que six mois dans un centre d'aide par le travail où il apprenait le métier de menuisier dans un atelier protégé. Bruno T. a également eu une scolarité médiocre. Il n'a pas obtenu son certificat d'étude. Il a été orienté en classe de $4^{\mathrm{e}}$ de transition et acheva sa scolarité à seize ans sans diplôme, ni formation. Il se fit embaucher comme cariste en entrepôt, puis barman, puis aide-boucher, peintre en bâtiment...

Plusieurs organes de presse, aux lendemains du procès, insistèrent sur les responsabilités de l'Éducation nationale et des filières où étaient cantonnés les élèves en échec scolaire. Rouge du 10 octobre 1975 dénonçait ces classes qui fabriquaient «à la chaîne » des frustrés, des paumés, des exclus. Le département de l'Oise comptait une forte proportion de jeunes sortant du système scolaire à seize ans sans aucun diplôme ni qualification.

7 De très petits délits sont à l'origine de leur prise en charge par la justice des mineurs à l'exception de Patrick. Yannick a commis un vol à l'âge de seize ans et demi. Le juge des enfants l'envoie dans un centre d'aide par le travail. Il n'y demeure que quelques mois, en est retiré à cause de troubles de comportement et est remis à ses parents. Lorsque le crime fut commis, il était soumis à la liberté surveillée jusqu'à l'âge de 20 ans pour ce vol, celui d'une mobylette. Michel avait volé l'un de ses premiers patrons dès l'âge de quinze ans, parce qu'il l'accusait de ne pas le payer assez. Bruno avait commis un vol chez l'une des clientes de son patron. Il s'enfuit du domicile familial et fut repris après une semaine. Livré à lui-même dans le Pas-de-Calais, un juge pour enfants estima que le foyer de Beauvais lui offrirait un encadrement approprié. À l'exception de Patrick, c'étaient de menus délits, des vols, qui étaient à l'origine de leur placement au foyer de Beauvais.

8 À Beauvais, Michel ne supportait pas l'ordre intérieur. Il en avait déjà fugué à plusieurs reprises. Bruno s'y plaisait. Il avait l'impression de connaître pour la première fois la liberté, loin de ce beau-père avec qui il ne s'entendait pas. Il en venait à oublier les orphelinats qu'il assimilait à des prisons, entourés comme elles de hauts murs et administrés par des règlements draconiens. Plus tard, il se confia à une assistante sociale : « Depuis l'âge de trois ans, j'ai comme des menottes aux poignets. » Le foyer ne conservait pas les jeunes au-delà de l'âge de dix-huit ans. Bruno avait demandé aux éducateurs s'il lui serait possible de demeurer au foyer une année supplémentaire. Il exprima ce vœu en novembre 1974, soit quelques mois avant l'échéance de sa majorité en mars 1975. Il essuya un refus qu'il vécut mal. Il était alors embauché dans une entreprise de carrosserie. Yannick ne fut placé au foyer qu'en novembre 1974, alors que Michel y était depuis le mois de mars et Patrick depuis le mois d'août.

\section{Un crime prémédité}

9 La question de la préméditation du crime commis à Liancourt fut au cœur du procès. Les chroniqueurs de presse furent quasiment unanimes pour constater que les jeunes avaient envisagé plusieurs jours à l'avance d'éliminer la victime qu'ils allaient détrousser puisqu'elle connaissait l'un d'entre eux. Le rapport de la chambre d'accusation fut entièrement construit pour démontrer cette préméditation. Les organes de presse partisans de l'application de la peine de mort insistèrent sur cette 
préparation, sur ce plan élaboré collectivement. Les récits du crime furent donc déterminés par cette circonstance aggravante, celle qui allait déterminer la sentence de mort pour Bruno T. Minute avançait une hypothèse supplémentaire. Parce qu'il pensait que l'on n'appliquait pas la peine de mort à des mineurs, Bruno T. aurait envisagé l'assassinat de Madame Boursier. Le journaliste Jean-Pax Mefret allait encore plus loin puisqu'il avançait une autre circonstance aggravante: un an auparavant, en novembre 1973, Bruno T. aurait déjà eu l'idée de s'en prendre à une vieille femme, projet abandonné, car elle demeurait trop près d'un commissariat.

10 C'est le mardi 19 novembre que cinq jeunes du foyer d'action éducative de Beauvais se réunirent dans la chambre de Bruno afin d'envisager un "mauvais coup » afin de se procurer de l'argent. Il y a là Bruno T., Patrick, Yannick, Michel et Patrick S., un comparse qui ne devait pas participer au crime final. Ils souhaitent fuir le foyer, partir en Italie ou en Hollande. Ils avaient partagé de bons moments lors d'un camp d'été à Chamonix. Partir à l'étranger, prendre la route comme le faisaient de nombreux jeunes à cette époque, leur faisait envie. Il leur fallait de l'argent et ils envisagèrent « un coup » pour s'en procurer rapidement. Yannick suggère le cambriolage d'une pharmacie ou la demeure d'un garde-chasse de la région d'Amiens. Patrick S. propose de dévaliser une station-service à Clermont de l'Oise. Ils se mettent d'accord sur ce projet qui nécessite de se procurer un véhicule.

11 Le lendemain, mercredi 20 novembre 1974, Patrick S., Yannick et Bruno parcourent les rues de Beauvais en quête d'une voiture à dérober. Ils en repèrent une près de la Caisse d'épargne. Le propriétaire survient et le vol du véhicule ne peut avoir lieu. En désespoir de cause, la petite bande écoute une nouvelle proposition de Bruno. Il propose de dévaliser une retraitée de Liancourt qui disposait « d'un million » d'économies et qui vit seule. C'était l'ancienne bouchère de la ville. Il connaissait la veuve Boursier, car elle était la grand-mère de l'un de ses camarades d'enfance. De temps en temps, il était allé couper du bois chez elle pour rendre service. C'est à ce moment que Bruno expliqua pour la première fois qu'il était impératif de tuer cette femme après l'avoir dévalisée, car elle le connaissait et risquait de le dénoncer. Ils acquiescèrent à l'exception de Patrick S. Il ne voulait pas participer à un meurtre. Il donna sa parole d'ancien louveteau de ne rien dire. Ce serment lui valut une année d'emprisonnement pour nondénonciation de crime. Les autres se répartirent les rôles. Bruno précisa qu'il avait eu le mérite d'apporter l'affaire et qu'il laisserait donc aux autres le soin de tuer la victime. Il suggérait de l'assommer dans sa cave, puis de pendre son corps pour faire croire à un suicide. Deux des jeunes gens se proposèrent pour étrangler la vieille dame. Michel ajouta qu'il saurait user d'un poignard, si c'était nécessaire. Cette version des faits fut celle de l'accusation. Dans son livre publié juste après le procès, Tuer les jeunes?, Antoine Marcilhacy niait la préméditation. C'est au cours de l'agression que Bruno T. aurait pris conscience du danger de son acte. De crainte d'être dénoncé, il aurait supprimé la vieille dame.

Le samedi 23 novembre était le jour fixé pour l'agression. Pendant la matinée, Bruno T. se rendit dans plusieurs bars et consomma du vin. Il rencontra une jeune femme à qui il proposa de l'emmener au cinéma dans la soirée. Il proposa donc à ses camarades de repousser l'agression au dimanche. Ils rétorquèrent qu'il était plus facile de quitter le foyer le samedi soir, car la surveillance y était exercée par une éducatrice. Le samedi, les jeunes du foyer avaient la permission de minuit. Bruno T. et Michel se rendirent aux Nouvelles Galeries et achetèrent des paires de gants. Nouvelles haltes dans les cafés de 
la ville, comme bien des jeunes en ce début de week-end. De retour au foyer, Bruno T. se fait remettre son livret de Caisse d'épargne par l'éducatrice. En fin d'après-midi, ils prennent un taxi pour Liancourt. Les quatre garçons arrivèrent dans la ville vers vingt heures trente et se firent déposer près de l'église.

Bruno T. mène l'équipe vers la demeure de la retraitée, impasse Niville. Les lumières sont éteintes dans le pavillon de briques roses, mais un poste de télévision est allumé. Ils escaladent le portail. Ils frappent à la porte, appellent la veuve qui hésite à ouvrir. "C'est Bruno ", indique celui qui est déjà venu chez la dame. Elle ouvre. Bruno T. se jette sur elle et ils s'engouffrent en la menaçant de leurs pistolets d'alarme. Michel demeure à l'extérieur pour faire le guet. Les jeunes gens réclament de l'argent. Elle sort son porte-monnaie et leur tend un billet de 100 francs en affirmant qu'elle n'a rien d'autre à la maison. Ils commencent à fouiller les meubles. Bruno T. trouve un chéquier dans l'un des tiroirs du buffet de la cuisine. Il exige de la vieille dame de leur faire un chèque d'un montant de 6000 francs. Madame Boursier tente de les amadouer et leur verse trois verres de vin. Elle est repoussée dans sa chambre à coucher par Yannick et Patrick. Ils découvrent quelques bijoux. Ils conduisent ensuite la vieille dame à la cave. Là, Patrick lui demande où se trouve son argent. Il la gifle violemment et commence à lui serrer le cou tandis que son complice lui donne des coups de poing au visage. Patrick ne parvient pas à étrangler la vieille dame et, épuisé, laisse son camarade le remplacer. À son tour, Yannick essaie de l'étrangler. Bruno T. descend et voit que ses camarades ne parviennent pas à tuer la vieille dame. Il remonte au rez-de-chaussée auprès de Michel pour lui prendre le poignard. Il revient dans la cave, donne un coup de pied dans le ventre de la victime, puis la poignarde près du cœur. Il s'acharne sur elle et la tue de treize coups de poignard. Avant de prendre la fuite, Yannick vole encore une montre et un transistor. Bruno T. enfouit le poignard dans un coin du jardin. C'est le début d'une fuite qui mène deux d'entre eux jusqu'à Amiens et les autres à Tourcoing. Bruno $\mathrm{T}$. et Patrick décident de franchir la frontière, vont à Gand, Amsterdam, Rotterdam, Coblence où ils se font arrêter le 26 novembre, avant d'être refoulés vers la France. Yannick et Michel avaient été arrêtés chez leurs parents le 25 novembre. Tous les quatre furent écroués dans la maison d'arrêt et de correction de Beauvais.

\section{Les assassinats de personnes âgées}

14 La justice française, depuis longtemps, sanctionnait sévèrement les assassinats de personnes âgées. Le 9 janvier 1975, Jacques Chancel recevait dans son émission de France Inter Claude Charmes. Il avait bénéficié d'une libération conditionnelle l'année précédente et avait publié une autobiographie, Le Maximum ${ }^{4}$. Claude Charmes, qui était lui aussi passé par les foyers de l'Éducation surveillée notamment Aniane ${ }^{5}$, était plus âgé que Bruno T. lorsqu'il fut condamné à mort le 24 avril 1960. Il avait vingt-huit ans, était jeune père de famille et séparé de son épouse. Il attendit plus de sept mois dans une cellule de condamné à mort la commutation de cette sentence en réclusion criminelle à perpétuité. Il avait étranglé une femme de soixante-sept ans pour lui voler sa pension trimestrielle de 900 francs.

15 Avant Claude Charmes, d'autres assassins de vieilles personnes avaient été sanctionnés de la peine de mort. Et ils n'avaient pas toujours été graciés. Dès la Libération, des meurtriers de personnes âgées sont fusillés. Jean Schmitt fut exécuté le 31 août 1946 pour avoir assassiné à coups de bêche une femme de soixante-treize ans près 
d'Angoulême. Il lui avait volé une somme d'argent et un morceau de lard. La même année Stanislas Walezewsky avoua quatorze meurtres de vieux fermiers commis pendant l'Occupation lors de vols et de cambriolages. Pour découvrir leurs économies, leurs lingots d'or, il les torturait et les massacrait à coups de serpette, de chenets, de bêches. De l'âge de vingt ans à celui de vingt-cinq ans, il avait parcouru le Lot-etGaronne, repérant les vieux couples d'agriculteurs isolés dans leur exploitation. On évoquait à son propos les «chauffeurs » de la Révolution. Il fut abattu par la police, l'année suivante, après son évasion de la prison de Montauban'.

Parmi les assassins d'anciens, les parricides suscitaient l'hostilité des jurés et de l'opinion. Un mineur figura parmi ces assassins de proches. Robert B., condamné à mort par les assises de la Seine, tua sa mère à coups de serpette, après sa libération d'une maison de correction. Il lui avait volé du linge et des vêtements en décembre 1946. Il bénéficia d'une grâce en 1948. Il n'avait alors que dix-neuf ans lors du crime. René Pons ne bénéficia pas de la mansuétude du général De Gaulle, car il fut guillotiné le 21 juin 1960. Il avait assassiné sa mère octogénaire avec la complicité de sa compagne ; ensuite, il avait fait brûler son corps dans la cheminée de sa demeure.

Plus de cinquante condamnations à mort sanctionnèrent des assassinats de personnes âgées de la Libération au procès de Bruno $\mathrm{T}$. Toutes ne furent pas exécutées. La jeunesse des auteurs est l'un des traits majeurs de cette criminalité. La cruauté accompagne fréquemment le crime. Une affaire assez ancienne, puisque jugée en 1958 à Angers par la cour d'assises des mineurs, comportait quelques ressemblances avec l'expédition des quatre jeunes du foyer de Beauvais. Trois jeunes de dix-sept ans étaient montés dans le train effectuant la liaison entre Quimper et Paris. Ils avaient projeté de dévaliser la recette de la gare d'une ville de province. Pour "s'entraîner ", ils assassinèrent un passager du train, un retraité de soixante-quatorze ans et lui volèrent 300 francs. Deux frères furent condamnés à vingt ans de travaux forcés. Celui qui avait porté les coups mortels fut condamné à mort ; il fut gracié le 23 octobre $1958^{7}$.

Les sentences les plus proches chronologiquement du procès de Bruno T. et de ses complices avaient été prononcées en 1973. Jean-Claude Bruckmann avait assassiné une femme de soixante-treize ans à coups de couteau. Sa première condamnation à mort avait été cassée. Rejugé, il avait été condamné à la réclusion criminelle à perpétuité par la cour d'assises de Nancy en octobre 1973. Robert Hennebert et Roger Davoine qui avaient égorgé un couple de vieillards pour les voler à Cambrai avaient été graciés en mars 1974.

\section{Le procès de Beauvais}

19 L'émotion avait été considérable à Liancourt aux lendemains du drame. "Odieux assassinat ", "lâche agression ", "bassesse d'un crime commis en pleine lucidité ", " meurtre abominable», " acte monstrueux », telles étaient les expressions du Bulletin paroissial de Liancourt de décembre 1974 rédigé par le curé André Gazeau qui s'efforçait pourtant de préserver les quatre jeunes du lynchage. Il s'interrogeait sur la responsabilité des parents dans ces déviances violentes, sur l'effet des films pornographiques ou meurtriers, sur les mauvais exemples donnés par les magazines licencieux. L'opinion locale était totalement hostile aux jeunes gens, comme le montraient les journaux locaux. 
20 À Beauvais, le procès devant la cour d'assises dura deux journées. Il se déroula à huis clos. Le procureur général réclama la réclusion criminelle à perpétuité pour Bruno et de longues peines pour ses complices. Le Monde donna quelques informations sur le fonctionnement du jury ${ }^{8}$. Malgré l'avis des trois magistrats professionnels, un premier vote des jurés écarte "l'excuse atténuante de minorité ». Elle avait été refusée, ce qu'autorisait le Code puisqu'au moment des faits, ils étaient âgés de plus de seize ans. Les peines prononcées pouvaient donc être les mêmes que celles encourues par un individu majeur. Les jurés allèrent plus loin que ce que souhaitaient les magistrats professionnels, magistrats du parquet comme du siège, ce qu'a relaté Robert Badinter. Ce fut la peine de mort pour Bruno, vingt ans de réclusion criminelle pour Yannick et Patrick et douze années d'emprisonnement pour Michel.

21 L'avocat de Bruno, maître Gervais, annonça qu'il allait déposer un pourvoi en cassation et il ajouta: «La décision a été rendue dans un climat passionnel. Vouloir faire un exemple n'est pas juger et l'esprit de vengeance n'a rien à voir avec la justice.» Bruno vécut plusieurs mois dans une cellule de condamné à mort de la maison d'arrêt d'Amiens. Bruno T. ne se rendait pas compte de la gravité de la sentence et était persuadé qu'il ne serait pas exécuté. Il s'apprêtait à supporter le délai de trois mois qu'il fallait à la cour de cassation pour se prononcer. Des hebdomadaires parvinrent à se procurer le réquisitoire du procureur et offrirent quelques indiscrétions sur le déroulement du procès. Bruno T. fut surpris d'être condamné à mort. À l'annonce du verdict, il protesta: "Vous n'avez pas le droit, je suis mineur. » Pendant le procès, il pensait ne risquer qu'un emprisonnement, au pire la perpétuité. Une réplique au président de la cour fut reproduite par Minute et Paris Match: "J'ai rien à dire maintenant, je vous raconterai après le verdict, quand vous m'aurez donné vingt ans.» Son attitude arrogante indisposa vraisemblablement les jurés. Il aurait également déclaré : «Oui, si c'était à refaire, je recommencerais ${ }^{9}$.» Cette froideur supposée, cette indifférence furent fréquemment citées par les partisans de la peine de mort. À leurs yeux, ce jeune homme d'un mètre soixante-quinze et de quatre-vingts kilos, n'était plus un enfant et ils partageaient le point de vue des jurés qui avaient repoussé l'excuse atténuante de minorité. C'était du moins l'opinion d'une fraction du public.

Durant le week-end qui suivit le verdict, la presse écrite et audiovisuelle exprima une certaine surprise face à la gravité de la sentence. Les organes de presse partisans d'une répression sévère des déviances insistaient sur l'horreur du crime commis. Le Journal du Dimanche du 5 octobre 1975 faisait dire aux habitants du village de naissance du principal accusé qu'ils l'auraient condamné à mort. Une boulangère espérait qu'il ne serait pas gracié et regrettait que les complices n'aient pas été condamnés à mort également. Mais dans les journaux télévisés, on pouvait aussi entendre des voisins, des copains présenter Bruno T. comme « un pauvre gosse, placé tout petit en orphelinat, qui n'avait pas toujours eu à manger ", et qui ne méritait pas une sentence aussi lourde. Les autorités religieuses catholiques et protestantes de Beauvais déplorèrent ce verdict de mort. Le Syndicat national des personnels de l'Éducation surveillée fut la première organisation de l'univers judiciaire à protester contre le verdict. Les journalistes recherchaient la date de la dernière exécution d'un mineur. Ils citaient fréquemment une exécution de janvier 1930 à Digne. En fait, il fallait remonter au 2 mai 1939, pour repérer la dernière exécution d'un mineur, condamné pour des crimes de droit commun. Quelques jours avant ses dix-huit ans, André Vittel avait subi le châtiment suprême, en public devant la prison Bonne Nouvelle de Rouen. Le Midi socialiste du 
mercredi 3 mai écrivait: «Il y a de longues années qu'un condamné aussi jeune fut exécuté. Si la grâce présidentielle n'intervint pas en faveur d'André Vittel c'est qu'il avait commis des crimes abominables. » Il avait frappé sa belle-sœur à coups de fer à repasser puis il l'avait égorgée avant d'étouffer son bébé de deux mois à l'aide d'un oreiller.

\section{Attente et agitations}

Aux lendemains de la condamnation, une intersyndicale des professionnels de la justice, plutôt classés à gauche, fit une déclaration. Le Syndicat de la magistrature, le Syndicat des avocats de France et les syndicats de l'enfance inadaptée CGT, CFDT, FEN indiquaient que cela faisait plus d'un quart de siècle qu'un jeune de moins de dix-huit ans avait été condamné à mort. La sécurité avait été invoquée par les plus hautes autorités de l'État et c'est en son nom que la sentence avait été prononcée. Le texte de la déclaration s'employait à éclairer "le sentiment général d'insécurité » en distinguant ses racines et causes économiques du phénomène de la délinquance qui était utilisée comme un bouc émissaire pour détourner l'attention de l'opinion publique des vrais problèmes de la société.

Pierre Blanchet avait recueilli dans Libération une première réaction auprès du porteparole du Syndicat de la magistrature réuni en convention à Vaucresson durant le week-end du 4 et 5 octobre 1975. Elle annonçait la ligne qui allait se déployer au sein des forces syndicales jusqu'à la commutation de la peine de Bruno T. Les communiqués successifs allaient s'en prendre à l'exploitation du sentiment d'insécurité par le ministre de l'Intérieur :

" (La sentence) nous paraît s'inscrire dans la stratégie du pouvoir et ministère de l'Intérieur en ce qui concerne le type de délinquance que l'on vise. Il y a un tel appel à la répression contre cette délinquance qu'on en arrive à ce que des jurés condamnent à mort un mineur. Il faut remonter à plusieurs dizaines d'années pour retrouver un tel exemple. Les jurés représentent quelque chose de l'opinion publique et celle-ci se modifie au gré des campagnes d'opinion. Bien entendu, les faits qui étaient reprochés à ce jeune étaient très graves, mais en prenant pour cible cette seule délinquance, on arrive au pire. Il y a deux délinquances, deux conceptions de l'ordre public. En faisant campagne sur un seul type de sécurité des Français, Poniatowski ne met en avant que certaines conceptions de l'ordre public. »

La Sofres publia un sondage le 24 octobre. $58 \%$ des Français étaient favorables à la peine de mort appliquée à des mineurs qui avaient tué pour de l'argent. Un mois plus tard, nouveau sondage du Centre d'éducation civique: $71 \%$ des Français étaient favorables au maintien de la peine de mort et les pourcentages s'élevaient à $90 \%$ pour son application aux cas de rapts d'enfants et $82 \%$ lors de prises d'otages. France-Soir publia un sondage de l'IFOP : 73 \% des Français exigeaient plus de sévérité à la justice. Sorj Chalandon dans Libération du 21 novembre commenta ce sondage en faisant une brève allusion à Bruno $\mathrm{T}$. :

«La France réclame du sang. Elle semble, à travers un mauvais rêve, baisser constamment le pouce au passage des condamnés, elle réclame des bagnes, des prisons à vie, des têtes de 17 ans, des expulsions pour tout ce qui ne lui ressemble pas, des brimades pour tout ce qui ne lui ressemble plus, elle semble hurler de joie et se repaître du sang de ceux qu'elle a enfantés. » 
thèmes d'une opposition de générations, les jeunes contre les vieux, d'une coupure entre l'univers des villes et des campagnes revinrent à plusieurs reprises sous la plume des commentateurs du verdict. Le Nouvel Observateur du 13 octobre 1975 accusait les jurés de Beauvais d'avoir déclenché une "guerre atroce " entre les générations, une société intolérante aux jeunes. Des jeunes qui se sentaient incompris, rejetés. Peu après le verdict de Beauvais s'ouvrit aux assises de Versailles le procès d'un jeune meurtrier, Jacky Pietkiewicz, ouvrier chez Simca. Il avait tué d'un coup de couteau l'assassin de son père, à l'occasion d'une reconstitution du drame. C'était une illustration de la loi du talion qui semblait inspirer de larges secteurs de l'opinion. Des pétitions signées par quarante mille personnes, des lettres de soutien contribuèrent à l'éclosion d'un large mouvement de solidarité envers le jeune prévenu. La presse qui souhaitait la mort pour Bruno $\mathrm{T}$. excusait le geste du fils qui avait vengé son père. Paris Match ( $\mathrm{n}^{\circ} 137718$ octobre 1975) comprenait ce jeune homme de vingt-cinq ans et dressait un portrait calamiteux de sa victime. Alain Grenouille avec un complice avaient été surnommés «les tueurs fous » et la comparaison avec les personnages du film de Stanley Kubrick Orange mécanique revint à plusieurs reprises sous la plume des chroniqueurs. Alain Grenouille était le neuvième enfant d'une famille pauvre de Cormeilles-en-Parisis. Très jeune, il connaît les centres d'Éducation surveillée, la prison. À la fin du mois de juillet 1971, Alain Grenouille avait alors dix-neuf ans et le compagnon de sa folle "équipée sauvage", Robert, seize ans. Ils volent plusieurs voitures. Ils sont armés de deux carabines. Alain Grenouille décide de tirer sur des cibles choisies au hasard. Ils abattent Victor Pietkiewicz qui revenait en vélomoteur de sa nuit de travail aux usines Simca. Les deux tireurs blessent un garde forestier, tuent un pompiste et sont arrêtés après un accident.

\section{Un traumatisme au sein de l'Éducation surveillée}

Le vendredi 10 octobre 1975, des élèves éducateurs et des personnels d'animation de l'Éducation surveillée firent un sit-in d'une heure et demie sur les marches du palais de justice à Paris. Ils furent soutenus par des avocats du Syndicat des avocats de France et du Mouvement d'action judiciaire. Une délégation fut reçue par le premier président de la cour d'appel. L'action était minoritaire et n'avait regroupé qu'une cinquantaine de personnels car le Syndicat national des personnels de l'Éducation surveillée réprouvait ce mode d'action.

Les éducateurs étaient inquiets de la réputation de leurs établissements qui, aux yeux de larges secteurs de l'opinion, étaient des pépinières de délinquants. Le jeudi 16 octobre 1975, le personnel du foyer d'action éducative de Beauvais tint une conférence de presse. Ils ne parvenaient plus à trouver des emplois pour les jeunes de l'Éducation surveillée qui leur étaient confiés.

En février 1976, les jours passaient dans l'attente de l'ultime décision du président de la République. Le 5 février, une trentaine d'éducateurs de l'Éducation surveillée se rendent au palais de justice de Paris en criant « Nous ne laisserons pas tuer Bruno ». Neuf d'entre eux s'enchaînent aux grilles du palais. Deux banderoles sont déployées : «Peine de mort = assassinat prémédité, légalisé, de sang-froid, gratuit, libéral et avancé ", "Non à la peine de mort, non à la détention ». Des membres du Mouvement d'action judiciaire, du Comité d'action des prisonniers et le chanteur Maxime Le Forestier leur

Revue d'histoire de l'enfance « irrégulière », 16 | 2014 
apportent leur soutien jusqu'avant que la police n'intervienne et les repousse place du Châtelet. D'après Libération, ces manifestants appartenaient au mouvement « Edukon ».

Le 15 janvier 1976, la chambre criminelle de la cour de cassation rejette le pourvoi. La veille le Syndicat national des personnels de l'Éducation surveillée (SNPES) avait publié une tribune dans Le Quotidien de Paris avec pour titre: "L'enjeu d'une tête». Il condamnait à nouveau le crime affreux commis sur une personne âgée mais dénonçait aussi le meurtre collectif, prémédité et légal qu'était la peine de mort. Le syndicat refusait l'argument de l'exemplarité de la peine puisque peu après le verdict condamnant Bruno, un crime analogue avait été commis dans une autre ville de France. Il réaffirmait sa foi dans l'homme, dans sa capacité à évoluer, à s'améliorer et donc s'opposait fermement à une sentence définitive. Les éducateurs indiquaient que l'insécurité provoquée par le chômage, les accidents, les agressions physiques, l'incertitude du lendemain était à l'origine du mouvement de colère de l'opinion lorsqu'un pas supplémentaire était franchi dans la sphère de l'intolérable. Ils insistaient sur les causes socio-économiques de la délinquance, des déviances et révoltes des jeunes.

Le crime commis par la bande de jeunes du foyer de Beauvais contribua à créer un climat d'hostilité en direction des structures de l'Éducation surveillée qui, elle-même, était parcourue de tensions et de débats sur les stratégies à mettre en œuvre. À Limoges, on assista à une levée de boucliers contre la création d'un nouveau centre pour jeunes délinquants. Ce devait être une structure publique d'orientation et d'éducation éducative en milieu ouvert. Elle suscita l'hostilité du secteur associatif et privé qui craignait la concurrence de ce nouvel établissement, des habitants du quartier résidentiel où elle devait s'installer et des élus locaux. Derrière les rumeurs et les arguments énoncés, il y avait la crainte irraisonnée des «jeunes", la peur face à cette population «dangereuse », la peur face à ces adolescents accueillis en « urgence » parce que les autres institutions n'en voulaient pas. Des rumeurs évoquaient près d'une centaine d'enfants alors qu'il n'était question que de l'hébergement d'une vingtaine. Une pétition circula contre le projet. Le préfet ouvrit une enquête.

L'affaire du crime de Liancourt et le verdict de Beauvais furent intégrés aux critiques $\mathrm{du}$ fonctionnement de la justice et du maintien de l'ordre en France et pour ainsi dire dilués dans une contestation globale plus ou moins radicale. L'annonce du verdict par Libération offrait un raccourci saisissant, sous un gros titre "La justice marche bien ». L'annonce de la condamnation à mort de Bruno, âgé de 17 ans, côtoyait une information sur la mise en liberté provisoire d'un dirigeant d'entreprise, inculpé d'homicide involontaire à la suite d'un accident de travail, après cinq journées de détention préventive. Gilles Millet qui consacrait de nombreux articles aux luttes des détenus, aux répressions policières, aux dysfonctionnements de la justice ne disait pas un mot de Bruno dans son éditorial. Il préférait fustiger une justice à deux vitesses, dure pour les petits et douce pour les puissants.

Bruno allait donc être "relégué » dans les pages intérieures des journaux dès le prononcé de sa condamnation. Cela annonçait le traitement qui allait être réservé à son cas durant les semaines pendant lesquelles il attendit la commutation de sa sentence de mort. Le quotidien Libération continuait d'apporter son soutien aux petits juges, membres du Syndicat de la magistrature, qui s'en prenaient à des industriels responsables de graves accidents de travail ou de catastrophes comme dans les mines du Nord de la France. La lecture de tous les exemplaires du quotidien pendant cette 
période, de novembre 1975 à janvier 1976, montre que l'affaire Bruno, mineur condamné à mort, n'était pas au cœur des préoccupations de l'équipe rédactionnelle ; probablement parce qu'elle présageait une grâce et parce que les schémas idéologiques de l'extrême gauche s'appliquaient plus facilement à des conflits de classe qu'à ce type de fait divers.

Rouge dénonçait la composition des jurys d'assises, juridiction appelée "frauduleusement populaire", composés de «citoyens honorablement connus dans leur mairie »... L'hebdomadaire fustigeait des jurys qui condamnaient sans preuves des accusés comme Pierre Goldmann, sur la foi de leur intime conviction. À propos de Bruno, les trotskistes écrivaient que les «classes de transition et classes de perfectionnement fabriquaient comme à la chaîne des frustrés, des paumés, des exclus... » et qu'il était " devenu à la fois victime et bourreau, instrument et objet de cette société de violence dont il est aussi le reflet ${ }^{10}$ ». La presse d'opposition se plut à citer des faits divers qui démontraient que le châtiment suprême n'avait rien empêché. Quinze jours après le verdict, à Nice, un jeune chômeur de dix-neuf ans, sans domicile fixe, se rendit chez une septuagénaire pour lui emprunter de l'argent. Il la connaissait car il avait logé dans son immeuble. Elle refusa. Furieux, il l'étrangla, puis lui porta huit coups de couteau.

\section{Les comités Bruno T.}

Un appel à la constitution d'un comité en faveur du jeune condamné à mort fut publié dans Libération le 18 octobre 1975. Le mardi 28 octobre plusieurs personnes se réunirent rue du Paradis à Paris, afin d'agir pour préserver son existence et pour lui apporter un réconfort moral. Elles souhaitaient mobiliser des personnalités afin de contrer les déclarations d'Alain Delon sur RTL qui avait traité Bruno T. de vermine. Un second comité vit le jour à Caen au début du mois de novembre. Ces structures de combat furent encouragées par Libération, le 8 novembre : «Ce combat participe réellement de la lutte contre le fascisme ordinaire qui se développe avec la violente campagne pour la "répression sans pitié" de la criminalité. » La presse régionale s'intéressa à l'initiative. La presse nationale, à l'exception de Libération, demeura quasiment muette. Quelle fut l'ampleur réelle de la mobilisation?

Le samedi 13 décembre 1975, Libération lança son premier supplément de quatre pages de petites annonces gratuites. Une rubrique "taulards " accueillait les demandes de correspondances et de contacts entre des prisonniers et des femmes. Au cœur de la rubrique figurait un encart : « Pour Bruno. Prison de Beauvais. Joyeux Noël, petit. » Un texte suivait signé « un prisonnier et des mères de familles qui ont honte de vivre dans un pays de pourriture qui est la France. » Il appelait à la solidarité et à l'aide financière en faveur du condamné à mort.

Il n'est pas évident non plus d'analyser la mobilisation internationale en faveur de Bruno T. Début février 1976, un " comité suédois Bruno » déposa à la présidence de la République une pétition de 5400 signatures demandant la grâce du jeune homme.

Le verdict de Beauvais intervint peu après le lancement d'une nouvelle campagne de l'Association française contre la peine de mort. Georgie Viennet, sa présidente, avait lancé le 1er octobre une campagne nationale et internationale pour l'abolition de la peine de mort dans le monde et pour des "peines de remplacement assumant pleinement la défense sociale ». Une pétition recueillit des milliers de signatures, 
chiffre certainement grossi par la sentence qui avait frappé Bruno T. Elles furent déposées le jeudi 27 novembre au Palais de l'Élysée. L'après-midi, au foyer du théâtre d'Orsay, les personnalités qui avaient toujours soutenu l'association donnèrent leur caution morale à la campagne. Afin de donner encore plus d'envergure à cette campagne, l'association organisa le 1er décembre une vente aux enchères publiques, "L'art vivant contre la peine de mort ». Un catalogue fut édité. Il présentait des œuvres d'artistes prestigieux .Il n'était pas fait mention de l'assassinat de Liancourt, mais il est indéniable que l'émotion suscitée par le verdict dans les milieux humanistes contribua au succès de cette nouvelle campagne.

\section{Les partisans d'une répression accrue}

Plusieurs organes de presse approuvèrent la sévérité des jurés de l'Oise, adoptant la même ligne de conduite que Le Journal du dimanche. Le Parisien libéré du groupe Amaury publia ce commentaire: "Mieux vaut espérer que la saine réaction amorcée dans le public et qui se traduit notamment par le verdict de Beauvais permettra aux Français de retrouver la sécurité. » Aux yeux du journaliste, les jurés de Beauvais avaient été l'expression de la majorité silencieuse qui «en a clairement assez de l'impunité des criminels, de leur remise en liberté systématique, assez des prisons quatre étoiles, assez de tout ce qui crée l'insécurité générale ${ }^{11} »$. Les appréciations de l'hebdomadaire d'extrême droite Minute, publié le 15 octobre, qui titrait « Oui à la guillotine » étaient très proches de celles du quotidien du matin : "Tout simplement, les honnêtes gens veulent vivre en sécurité. Et ils ont le sentiment justifié que la justice, telle qu'elle est appliquée actuellement, n'est pas suffisamment rigoureuse pour la leur garantir. " Le journaliste Frédéric Brémont s'en prenait au laxisme criminel, aux prisons "pensions de familles ", de "véritables passoires » où les peines n'étaient pas effectuées jusqu'à leur terme. Il estimait que les « crapules» devaient être «neutralisées »: «les tueurs doivent être tués ». À ses yeux, Bruno était un monstre qui ne devait pas bénéficier d'une grâce. Invité au « journal inattendu » de la station de radio RTL, Alain Delon eut des mots très durs à l'égard du jeune Bruno. Paris Match, le 25 octobre, rapportait ses propos :

«Cela, c'est de la vermine et la vermine il faut l'écraser... Ces gens-là n'ont pas le droit de vivre... Que mon fils me dise qu'il a fait la même chose, je vous affirme que je ferai justice moi-même et que je le tuerai de mes propres mains, car il ne serait plus mon fils.»

Monsieur Taron, le père de Luc Taron assassiné par Lucien Léger, animait une Ligue contre le crime et pour l'application de la peine de mort. Il organisait des campagnes de pétitions pour faire appliquer cette sentence. Jean-François Chaigneau dans Paris Match posait en introduction d'un article occupant deux pleines pages la question suivante: «Faut-il guillotiner Bruno?»

41 Dès le dimanche soir, Jean Lecanuet, garde des Sceaux, commenta le verdict au journal télévisé. Il évoqua l'insécurité que l'opinion populaire en colère ne supportait plus. Cette dernière exigeait de la rigueur et le ministre disait entendre ce message du pays : « je demande donc de la rigueur ». Ce discours fut salué par Minute (8 octobre 1975): "Le ministre de la Justice commence à parler comme Minute écrit. " Jean Lecanuet disait souhaiter que des peines sévères soient appliquées aux " crimes odieux ». Cet alignement du garde des Sceaux sur les positions répressives de Michel Poniatowski, 
ministre de l'Intérieur, fit l'objet de bien des sarcasmes. Une caricature de Solo dans Libération du 17 octobre 1975 avait pour titre: «Lecanuet: dents blanches, haleine fétide. » Le dessinateur avait placé une guillotine au milieu de sa dentition, crispant ce sourire qui avait fait le succès du candidat centriste aux élections présidentielles de 1965.

Le 15 janvier 1976, la cour de cassation rejeta le pourvoi. La presse parisienne ne commenta guère la décision de la haute juridiction. Le 21 janvier, Le Monde publia une tribune signée par Hubert Haddad, écrivain et animateur d'un foyer de jeunes à SaintMaur-des-Fossés. C'était un cri de colère " contre une barbarie moyenâgeuse ", titre qui avait été choisi par la rédaction. Il se prononçait évidemment contre la peine de mort. Le grand quotidien du soir n'offrait aucun commentaire sur la situation du jeune homme, en attente de la grâce présidentielle.

\section{En attendant la grâce, le procès d'Abdellaziz A. et le crime de Patrick Henry}

Le rapt du petit Philippe Bertrand à Troyes fut évoqué pour la première fois dans Libération le 2 février 1976 et dans Le Monde du même jour. Âgé de sept ans, l'enfant avait été enlevé à la sortie de son école de Pont-Sainte-Marie le vendredi 30 janvier vers midi. Moins d'une heure après, le ou les ravisseurs avaient téléphoné à la famille pour réclamer une rançon d'un million de francs. Le lendemain, la presse indiquait que le ravisseur, écouté lors d'une conversation avec le père de l'enfant, ne s'exprimait pas avec les mots du Milieu. Il était poli, déférent, son langage était celui «d'un petit employé ", selon les policiers. L'information sur le rapt n'occupait que quelques lignes dans les deux quotidiens, mais il en était question chaque jour. Cette répétition de brèves et d'articles contribua à la montée de l'angoisse. Une intervention de Jean Lecanuet, garde des Sceaux, dans l'émission C'est-à-dire d'Antenne 2 du 4 février était lourde de menaces et annonçait des châtiments impitoyables: "J'ai recommandé au parquet la plus grande fermeté. [...] Pour ces criminels, qui sont des calculateurs odieux, qui n'ont aucun respect de la vie, j'irai jusqu'à recommander la peine de mort. »

Le samedi 7 février, Libération commentait un verdict de condamnation à la réclusion à perpétuité d'un mineur qui avait assassiné un chauffeur de taxi pour lui voler sa recette. Ce lad de Maisons-Laffitte, Abdelaziz A. n'avait que seize ans et dix mois à l'époque des faits. Comme Bruno T. et ses amis, il avait prémédité son forfait et s'apprêtait à récidiver, avec un complice, lorsqu'il avait été arrêté. L'article de première page, titré « Dans la ligne de Beauvais » se concluait ainsi :

«Le pire, maintenant qu'un mineur a été condamné à mort, c'est qu'un verdict comme celui-ci va paraître clément. On commence déjà à dire qu'il n'y a pas de nouvelle affaire Bruno. Une telle interprétation donne une idée du contexte répressif actuel, surtout à l'égard des jeunes délinquants. »

L'auteur de ce court article était probablement Gilles Millet, car l'argumentation était comparable à celle qui avait suivi le verdict d'octobre 1975. Libération voulait se démarquer des textes "larmoyants et moralisateurs » qui avaient suivi le verdict de Beauvais, selon l'expression de Dominique Frot. La peine de la réclusion à perpétuité était aux yeux de cette équipe de journalistes une autre mort et le sort des 30000 détenus des prisons françaises devait retenir l'attention, autant sinon plus que le cas exceptionnel du jeune mineur promis au châtiment suprême. Le Monde du 6 février 1976 
précisa que le procès du jeune marocain s'était déroulé dans un climat de profond malaise car tous les acteurs du procès devant la cour d'assises des mineurs des Yvelines avaient en tête la sentence prononcée à l'encontre de Bruno T. En présence d'un crime d'une particulière atrocité, les observateurs s'interrogeaient sur l'attitude des jurés. La récidive était un facteur aggravant mais le substitut Monteils ne réclama aux jurés que la réclusion criminelle à perpétuité, arguant que le «degré d'atrocité était moindre comparé à l'agonie de la vieille dame de l'Oise». Le jeu de miroirs entre les deux affaires était donc dans tous les esprits et le verdict de Versailles pouvait être lu de différentes manières : verdict clément pour un mineur récidiviste coupable d'un crime atroce, donc qui laissait augurer une clémence présidentielle à l'égard d'un autre mineur ou bien crime moins grave que celui commis par Bruno T. ce qui justifierait le respect de l'échelle des peines et l'application d'une sentence plus sévère à son égard, en l'occurrence la mort.

Un communiqué du Palais de l'Élysée annonça la grâce du jeune Bruno T. le 11 février 1976 en début de soirée. Son avocat, maître Gérard Gervais avait été reçu pendant trois quarts d'heure la veille par le président. L'attente dans une cellule de condamné à mort était donc terminée. Il est difficile de hiérarchiser les raisons qui incitèrent le président Valéry Giscard d'Estaing à le gracier, en vertu de l'article 17 de la Constitution. C'était le premier condamné à mort du septennat et certains disaient qu'une tradition voulait que le premier condamné à mort d'un septennat soit toujours gracié. L'image libérale que le président souhaitait se donner aurait été entachée par une première exécution, peu de temps après celles commises en Espagne par le régime franquiste finissant. Dans Libération, Dominique Frot, dans des articles du 12 et du 13 février, s'interrogeait sur le credo du président à l'égard de la peine de mort. Aurait-il gracié Bruno contre les $58 \%$ de Français qui étaient partisans de son exécution, si le pays s'était trouvé à la veille d'une échéance électorale ? Les Français étaient répressifs et encouragés à l'être par le ministre de l'Intérieur et par une partie de la presse. Le président Valéry Giscard d'Estaing n'avait jamais exprimé son point de vue sur la question. Certes, le candidat, en avril 1974, avait déclaré qu'il avait "naturellement, comme chacun, une aversion profonde pour la peine de mort ", qu'il était nécessaire de recueillir des avis pour ne pas se prononcer à la légère. Le journaliste exprimait ses craintes pour l'avenir lorsque des condamnés à mort seraient d'une "autre trempe " que Bruno; des criminels calculateurs ou preneurs d'otages pour qui le garde des Sceaux Lecanuet conseillait de faire subsister la peine de mort dans l'échelle des peines, dans une déclaration à Europe $\mathrm{n}^{\circ} 1$ du 11 février 1976. Philippe Boucher, dans LeMonde du 13 février, partageait ces appréhensions : «Il ne ferait pas bon être jugé ces jours-ci pour un rapt d'enfant! Le talion y a le pas sur la loi.»

\section{L'explosion de haine envers Patrick Henry}

Sept jours après le communiqué élyséen, un petit encart titré «Philippe est mort » figurait sur la première page de Libération :

«Le cadavre du petit Philippe Bertrand enveloppé dans un drap et une couverture a été découvert en début de soirée dans un hôtel de Troyes. Cette chambre était occupée par le suspect que les policiers ont entendu récemment durant 48 heures. L'homme, Patrick Henry, a été immédiatement appréhendé. » 
Dès le lendemain, le 19 février, le quotidien avait compris que la peine de mort allait à nouveau être au cœur des débats lors du procès de Patrick Henry. Il titrait : «Rapt de Troyes: campagne pour la peine de mort. Crime contre crime. La répression passe avant l'otage. » Serge July consacra un éditorial au dénouement du rapt de Troyes. Il s'en prit aux partisans du "meurtre d'État", notamment les deux ministres de la Justice et de l'Intérieur. Michel Poniatowski avait clairement exprimé son choix: " J'espère que la justice passera rude et vite.» Patrick Henry était déjà virtuellement condamné à mort par les plus hautes autorités de l'État et par une partie de la presse. Dans le Journal du Dimanche René Barjavel fut explicite: "Assez de pitié pour les monstres $^{12}$. " Philippe Boucher écrivit dans Le Monde du 20 février 1976 : «Où est l'État quand le langage de ses ministres ne se distingue en rien de celui qu'on prête à l'homme de la rue, dominé par la peur et l'esprit de vengeance? » Durant un mois on assista à une véritable "tempête justicière", à un "exorcisme collectif", selon les expressions de Pierre Viansson-Ponté dans Le Monde du 29 février 1976. Le vendredi 20 février une caricature de Daulle en première page de Libération montrait un détenu au costume rayé de bagnard en train de clouer les bois d'une guillotine. La légende était la suivante : « De la grâce de Bruno T... à l'hystérie de Troyes. Après la grâce de Giscard, l'Administration pénitentiaire décide d'apprendre au jeune Bruno T. le métier de menuisier.»

La réclusion criminelle à perpétuité de Bruno T. fut commuée en vingt années de réclusion criminelle le 17 février 1984.

\section{NOTES}

1. Nouvel Observateur, 13 octobre 1975.

2. MARCILHACY Antoine, Tuer les jeunes ? Paris, Tema-Éditions, 1976.

3. Toutes les informations sur les antécédents des mineurs et sur l'affaire sont issues du dossier d'instruction.

4. CHARMES Claude, Le Maximum, Paris, Stock, 1974.

5. BouRquin Jacques, « René Biard, Claude Charmes, deux "enfants de justice" pendant la guerre », Revue d'histoire de l'enfance "irrégulière», $\mathrm{n}^{\circ} 3,2000$, mis en ligne le 30 avril 2007.

6. Sur l'affaire Walezewsky on peut lire DEHAYE Bruno, Les nouvelles affaires criminelles de l'Aisne, De Borée, 2010, 321 p. Le récit est quelque peu romancé mais il s'appuie sur le dossier de procédure.

7. [http://guillotine.voila.net/].

8. Le Monde, 11 octobre 1975.

9. Paris Match, $\mathrm{n}^{\circ} 1377$ du 18 octobre 1975 ; Minute, $\mathrm{n}^{\circ} 705$ du 15 octobre 1975.

10. Rouge, 15 octobre 1975.

11. Le Parisien libéré, 5 octobre 1975. 


\section{RÉSUMÉS}

Quelques jours avant l'arrestation de Patrick Henry, auteur du kidnapping et de l'assassinat d'un enfant, un adolescent, mineur aux yeux de la justice, fut gracié par le président Giscard d'Estaing. Sa peine de mort prononcée fut commuée en réclusion à perpétuité. Avec trois autres mineurs d'un centre de l'Éducation surveillée, il avait assassiné une vieille dame pour la voler. Bruno T. fut le dernier mineur condamné à mort sous la $\mathrm{V}^{\mathrm{e}}$ République. L'article offre des éléments de procédure concernant cette affaire, le contexte sécuritaire de cette époque, l'émotion et les réactions provoquées par le verdict. Il évoque également les actions de protestation contre la peine de mort et de solidarité envers ce mineur délinquant.

A few days before the arrest of Patrick Henry --who had kidnapped and killed a child--, a teenager, who was regarded as a minor by the law, was granted a reprieve by President Giscard d'Estaing. His death sentence was thus turned into life imprisonment. With three other under-18s from the centre of controlled education, he had murdered an old lady in order to rob her. Bruno T. was the last minor youth to be sentenced to death during the 5th Republic. This article purports to recall some procedural information about the case, but also to deal with the security context of the time, the emotion and reactions triggered by the verdict. It also tackles the various protests against death penalty that took place in those days as well as the actions meant to show support to this juvenile delinquent.

\section{INDEX}

Mots-clés : histoire, adolescent criminel, délinquance juvénile, France, XXe siècle, éducation surveillée, peine de mort, opinion publique

Keywords : history, death penalty, criminal teenager, juvenile delinquency, public opinion, France, XXth century, state institution for juvenile offenders

\section{AUTEUR}

\section{JEAN-CLAUDE VIMONT}

Maître de conférences, membre du GRHis (EA3831) à l'université de Rouen et du comité de rédaction de Criminocorpus. 